Article

\title{
Arcanobacterium haemolyticum Phospholipase D Enzymatic Activity Promotes the Hemolytic Activity of the Cholesterol-Dependent Cytolysin Arcanolysin
}

\author{
Patrick S. Gellings and David J. McGee* \\ Department of Microbiology and Immunology, Louisiana State University Health Sciences Center-Shreveport, \\ 1501 Kings Highway, P.O. Box 33932, Shreveport, LA 71130-3932, USA; pgelli@lsuhsc.edu \\ * Correspondence: dmcgee@lsuhsc.edu
}

Received: 26 April 2018; Accepted: 18 May 2018; Published: 23 May 2018

check for updates

\begin{abstract}
Arcanolysin, produced by the human pathogen Arcanobacterium haemolyticum, is a cholesterol-dependent cytolysin. To mediate the pore-formation process, arcanolysin is secreted by A. haemolyticum and then must interact with cholesterol embedded within a host membrane. However, arcanolysin must compete with membrane components, such as the phospholipid sphingomyelin, to interact with cholesterol and form pores. Cholesterol forms transient hydrogen bonds with the extracellular portion of sphingomyelin, shielding cholesterol from extracellular factors, including arcanolysin. A. haemolyticum also produces a sphingomyelin-specific phospholipase $\mathrm{D}$, which removes the choline head from sphingomyelin, leaving cyclic-ceramide phosphate and eliminating the potential for cholesterol sequestration. We hypothesized that the enzymatic activity of phospholipase D decreases sphingomyelin-mediated cholesterol sequestration and increases cholesterol accessibility for arcanolysin. Using purified arcanolysin and phospholipase D, we demonstrate that the enzymatic activity of phospholipase D is necessary to promote arcanolysin-mediated hemolysis in both time- and concentration-dependent manners. Phospholipase D promotion of arcanolysin-mediated cytotoxicity was confirmed in Detroit 562 epithelial cells. Furthermore, we determined that incubating phospholipase D with erythrocytes corresponds with an increase in the amount of arcanolysin bound to host membranes. This observation suggests that phospholipase D promotes arcanolysin-mediated cytotoxicity by increasing the ability of arcanolysin to bind to a host membrane.
\end{abstract}

Keywords: sphingomyelinase; cholesterol-dependent cytolysin; Arcanobacterium haemolyticum; enzyme mutation; protein purification

Key Contribution: The two known virulence factors of Arcanobacterium haemolyticum, arcanolysin and phospholipase D, synergistically form pores within eukaryotic membranes, affecting membrane integrity and cell viability.

\section{Introduction}

Arcanobacterium haemolyticum is a Gram-positive bacterium that is a causative agent of pharyngitis and wound infections in humans [1-4]. Little is known regarding the lifecycle and pathogenesis of A. haemolyticum despite its designation as an emerging pathogen by the Centers for Disease Control and Prevention over 20 years ago [1]. A. haemolyticum predominately infects adolescents and is the causative agent in approximately 240,000-480,000 cases of pharyngitis worldwide each year [1-4]. To date, only two A. haemolyticum putative virulence factors have been partially characterized: Arcanolysin (ALN) and phospholipase D (PLD).

ALN is a member of the pore-forming cholesterol-dependent cytolysin (CDC) family [5,6]. Over 50 different CDCs have been identified, predominately from Gram-positive bacteria [6]. The steps 
involved in the CDC pore-formation process are well understood: monomer binding, oligomerization, $\beta$-sheet formation and insertion for final pore formation and membrane disruption [7]. As their name implies, nearly all members of the CDC-family, including ALN, rely on interactions with membrane cholesterol to mediate the pore formation process [5,7]. Apart from the knowledge that ALN has considerable specificity for human erythrocytes over rabbit, ovine or bovine cells and a dependence on membrane cholesterol for pore-formation, very little is known regarding neither the biochemistry of ALN nor its role in A. haemolyticum pathogenesis [5].

The other A. haemolyticum virulence factor, PLD, is understood to be universally produced by all $A$. haemolyticum isolates [8]. Bacterial phospholipase $\mathrm{D}$ enzymes commonly hydrolyze both sphingomyelin (SM) and phosphatidylcholine (PC) and contain a His-X-Lys-X4-Asp (HKD) motif within the catalytic site [9]. A. haemolyticum PLD, however, does not contain the traditional HKD motif and instead causes the unique cyclization of SM or lysophosphatidylcholine (LPC), while having minimal effect on PC [10]. Interestingly, A. haemolyticum PLD is most similar to a series of Corynebacterium spp. phospholipase D enzymes as well as a multitude of fungal pathogens, most of which have received little to no interest from the scientific community. As a result of its unique properties, A. haemolyticum PLD converts SM or LPC into choline and either cyclic-ceramide phosphate (CCP) or cyclic-phosphatidic acid (CPA), respectively [10]. The ramifications of its unique biochemistry have not been fully investigated, but preliminary studies suggests PLD may impact bacterial adherence and invasion, host plasma membrane lipid raft formation and host cell necrosis [8].

Various membrane lipids are known to interact with one another within a host membrane. One such example exists between cholesterol and SM. Cholesterol molecules are embedded within a membrane but display a hydroxyl group to the outside environment [11]. Similar to cholesterol, the majority of a SM molecule is membrane-embedded but presents a hydrophilic choline head group to the extracellular environment [11]. The hydroxyl group of cholesterol and a portion of the hydrophilic head of SM form a transient hydrogen bond, creating an "umbrella-like" covering over cholesterol [11]. This SM covering shields cholesterol from extracellular factors, including ALN [11]. Removal of the choline head of SM eliminates the possibility of cholesterol-SM interactions, thus increasing the pool of accessible cholesterol [12]. Based on the cholesterol-dependence of ALN, the substrate specificity of PLD, and the cholesterol-SM interaction, we hypothesized that the two A. haemolyticum virulence factors may work in tandem to promote ALN pore-formation.

Using purified PLD or ALN and human erythrocytes, we demonstrate that the enzymatic activity of PLD promotes ALN-mediated hemolysis in both time- and concentration-dependent manners. Further, we observed an increase in ALN-membrane binding as a result of incubating erythrocytes with PLD. Overall, this study demonstrates a previously unidentified synergistic relationship among the two known virulence factors of $A$. haemolyticum: ALN and PLD.

\section{Results}

\subsection{A. haemolyticum Produces at Least Two Products Which Contribute to Hemolytic Activity of Bacterial Supernatants}

A diagnostic hallmark of $A$. haemolyticum is its strong $\beta$-hemolysis when grown on agar plates supplemented with human blood [1]. The size of the hemolytic rings suggests that one or more factors secreted by A. haemolyticum possess hemolytic activity.

Analysis of the A. haemolyticum genome suggests the presence of multiple, putative virulence factors, among which includes two factors that specifically target host membrane integrity: a sphingomyelin-specific phospholipase D (PLD) [8,10] and the CDC named ALN [5]. To measure the effect ALN and PLD have on the hemolytic capabilities of $A$. haemolyticum, supernatants were isolated from WT, $\Delta p l d, \Delta a l n$, and the corresponding complemented strains of A. haemolyticum and hemolytic activity was assessed (Figure 1). WT A. haemolyticum supernatants lysed $46.3 \%$ of erythrocytes in the assay while supernatants from the $\Delta a l n$ and $\Delta p l d$ strains lysed $17.5 \%$ and $3.1 \%$ of the erythrocytes, respectively (Figure 1). Complementing either mutant strain restores hemolysis, though not to the level 
of WT A. haemolyticum (Figure 1) which may be due to issues such as titration of limited regulatory factors or the fact that mutants were rescued with a multi-copy plasmid. These data suggest that both ALN and PLD contribute to hemolysis by the bacterium. Attempts to construct a $\Delta$ pld $\triangle a l n$ double mutant have so far been unsuccessful.

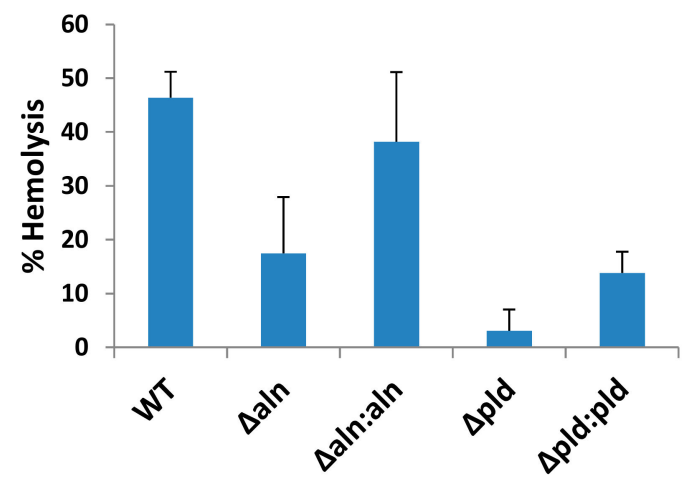

Figure 1. Absence of PLD or ALN decreases A. haemolyticum hemolysis levels. Overnight cultures of ATCC 9345 A. haemolyticum, $\Delta a l n, \Delta$ pld or their complements were centrifuged and culture supernatants were added to $2 \%$ human erythrocytes for $30 \mathrm{~min}$ at $37^{\circ} \mathrm{C}$. Samples were then centrifuged and the $\mathrm{OD}_{415}$ of the supernatants were measured and used to calculate the $\%$ hemolysis of each treatment. Results are representative of at least three independent experiments conducted in triplicate with error bars representing standard deviation of the mean.

\subsection{Purified His-ALN or His-PLD Is Sufficient to Cause Hemolysis of Human Erythrocytes}

When purified, CDCs, including ALN, are potent hemolysins [5-7]. However, it was previously reported that purified $A$. haemolyticum PLD contains no innate hemolytic activity by itself [8]. The observation that PLD does not contain hemolytic activity seemingly contradicts our data (Figure 1), which suggests PLD contributes to the hemolytic activity of $A$. haemolyticum supernatants. This apparent contradiction might be due to insufficient concentrations of purified PLD in prior experiments or its requirement of an unknown cofactor. To verify the hemolytic activity of PLD and ALN, $\mathrm{His}_{6}$ tagged recombinant ALN and PLD (His-ALN and His-PLD) were purified and the hemolytic activities of the individual proteins were measured against human erythrocytes. After 30 min incubation, sufficient concentrations of either His-ALN or His-PLD were determined to induce hemolysis of human erythrocytes, though the minimum concentration of His-ALN ( $20 \mathrm{ng} / \mathrm{mL})$ was approximately 1000 -fold lower than the minimum concentration of His-PLD necessary to cause hemolysis $(20 \mu \mathrm{g} / \mathrm{mL})$ (Figure 2).
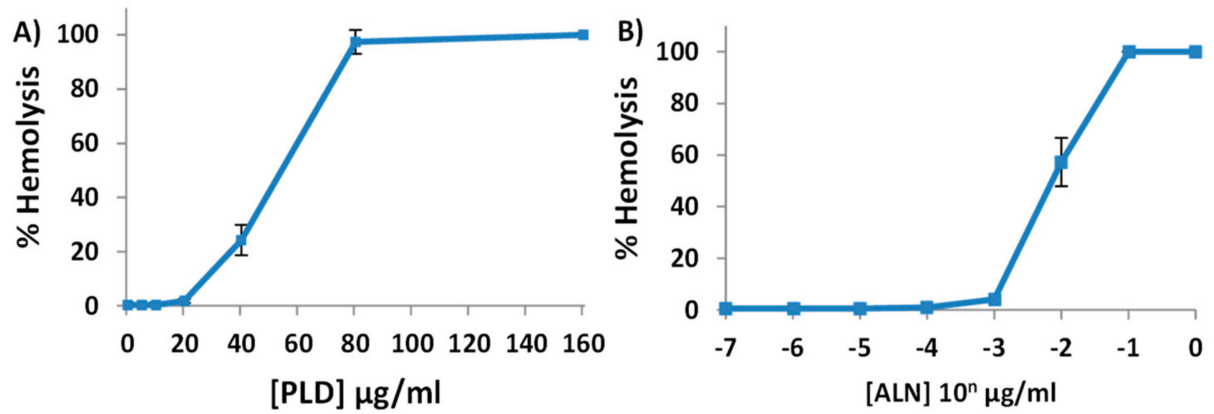

Figure 2. Purified PLD or ALN from A. haemolyticum is sufficient to induce hemolysis of human erythrocytes. Various concentrations of purified His-PLD (A) or His-ALN (B) were suspended in $1 \times$ PBS and added to $2 \%$ human erythrocytes for $30 \mathrm{~min}$ at $37{ }^{\circ} \mathrm{C}$. \% hemolysis was then determined as outlined in Figure 1. Results are representative of three independent experiments conducted in triplicate with error bars representing standard deviation. 
As a negative control, $H$. pylori RocF was similarly purified and subject to a similar concentration-dependent hemolysis assay [13]. Regardless of the concentration of RocF used, no hemolysis was observed (data not shown). Thus, either ALN or PLD are sufficient to cause hemolysis individually as purified proteins and may contribute to the hemolytic activity of A. haemolyticum supernatants.

\subsection{A. haemolyticum PLD Promotes ALN-Mediated Hemolysis in Both Time- and Concentration- Dependent Manners}

It was previously established that $A$. haemolyticum PLD contains sphingomyelinase activity [10]. Because of the sphingomyelin-mediated sequestration of cholesterol [14] and the dependence ALN has on cholesterol [5,7], we hypothesized that exposing human erythrocytes to PLD would increase the amount of accessible cholesterol, measurable by an increase in ALN-mediated hemolysis, in response to longer exposure to PLD. Sub-hemolytic concentrations of ALN were added to human erythrocytes for $30 \mathrm{~min}$ at $22^{\circ} \mathrm{C}$, followed by the addition of sub-hemolytic concentrations of PLD. In this order, ALN followed by PLD, no observable increase in hemolysis was detected (Figure 3A).

However, when the reciprocal experiment was carried out, incubating human erythrocytes first with PLD followed by ALN, ALN-mediated hemolysis greatly increased in response to higher concentrations (Figure 3B) or longer incubations of PLD (Figure 3C). Together, these experiments demonstrate that PLD promotes ALN-mediated hemolysis in an order-dependent fashion.
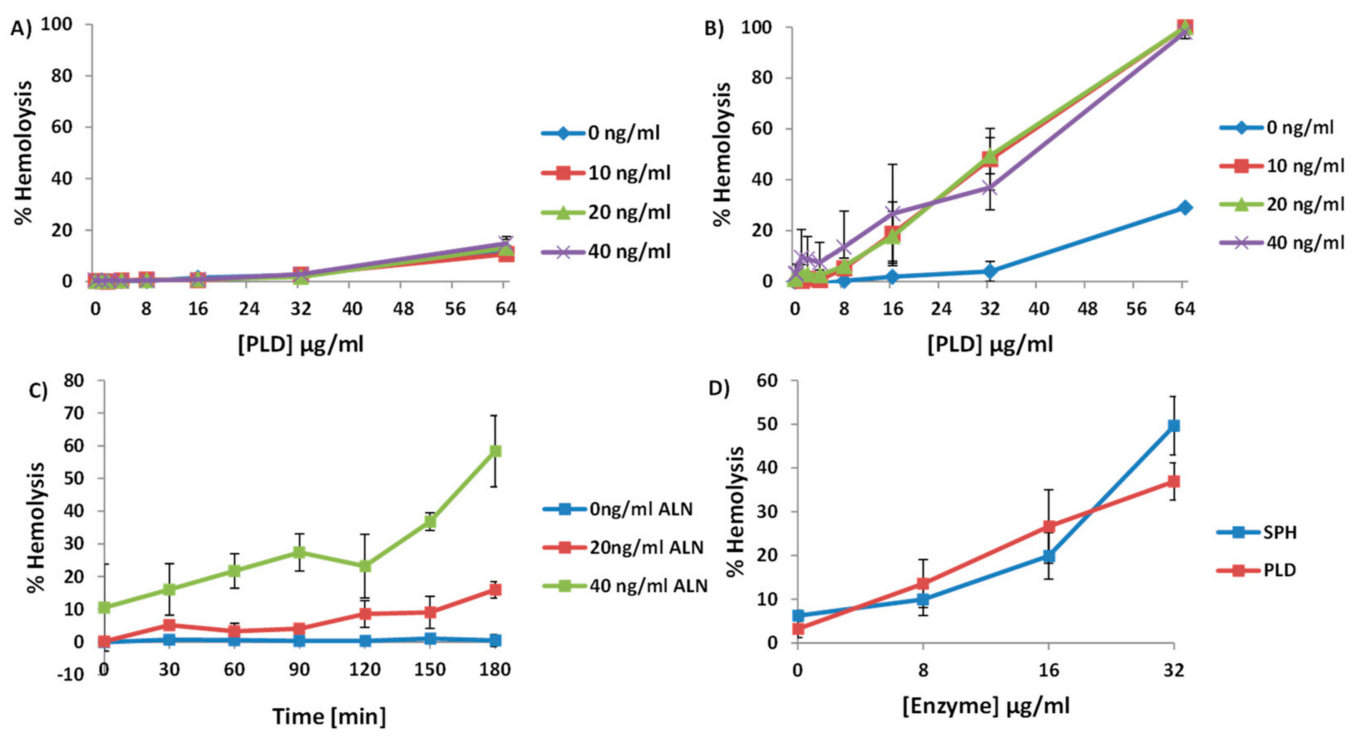

Figure 3. Pre-incubating human RBCs with PLD promotes ALN-mediated hemolysis in time- and concentration-dependent manners. (A) $0-40 \mathrm{ng} / \mathrm{mL}$ ALN was incubated with $4 \%$ human erythrocytes for $30 \mathrm{~min}$ at room temperature, followed by the addition of $0-64 \mu \mathrm{g} / \mathrm{mL}$ of PLD for an additional $30 \mathrm{~min}$, but little to no detectable hemolysis was observed. (B) When the reciprocal experiment was performed, erythrocyte incubation with PLD followed by the addition of ALN, there was a significant increase in hemolysis. (C) Equal parts $4 \%$ erythrocytes and $4 \mu \mathrm{g} / \mathrm{mL}$ PLD were incubated at room temperature for between 0 and $180 \mathrm{~min}$. At the end of each time point, equal parts PLD-treated erythrocytes and either 0,20 , or $40 \mathrm{ng} / \mathrm{mL}$ ALN were incubated together for an additional $30 \mathrm{~min}$. Each experiment was conducted three times. (D) Purified SPH or PLD were incubated with erythrocytes in a similar manner as previously mentioned, followed by the addition of $40 \mathrm{ng} / \mathrm{mL}$ ALN. Both SPH and PLD were able to promote ALN-mediated hemolysis in a similar manner. Results are representative of at least three independent experiments conducted in triplicate with error bars representing standard deviation of the mean. 


\subsection{An Increase in ALN-Mediated Hemolysis Is Not Dependent upon the Generation of Cyclic-Ceramide Phosphate}

In order to evaluate if the increase in ALN-mediated hemolysis is dependent upon eliminating the ability of SM to sequester cholesterol or is dependent on the generation of the unique cyclic ceramide derivative by PLD, we purified a recombinant form of sphingomyelinase D (SPH) produced by Bacillus anthracis [15]. SPH is well-characterized biochemically and converts sphingomyelin to phosphocholine and ceramide; no cyclic products are generated [15]. Similar to the experiments done in Figure 4, we incubated human erythrocytes with increasing concentrations of SPH followed by the addition of sub-hemolytic concentrations of ALN. Incubating erythrocytes with SPH led to an increase in ALN-mediated hemolysis in a concentration-dependent manner (Figure 3D).

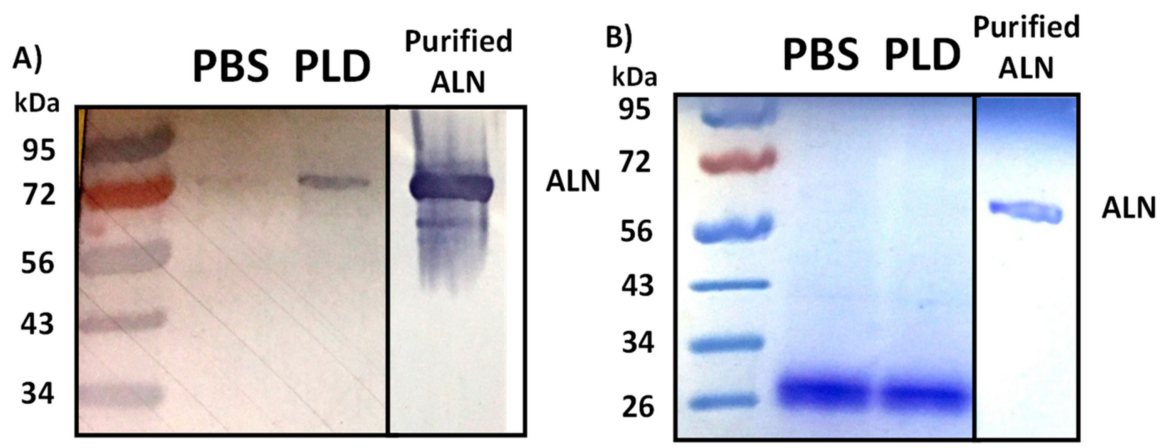

Figure 4. Analysis of ALN-binding in response to PLD treatment of whole human blood. (A) Whole human blood was treated with PBS only or PBS containing $1 \mu \mathrm{g} / \mathrm{mL}$ PLD for $60 \mathrm{~min}$ at $37^{\circ} \mathrm{C}$ with gentle agitation. PLD-treated samples were washed with PBS, chilled on ice, and equal parts blood and ice-cold ALN were incubated on ice with gentle agitation for $60 \mathrm{~min}$. Samples were washed three times with $1 \times$ PBS, suspended in loading buffer, and examined via Western blot using an anti-ALN antibody as described in Materials and Methods. Lane 1: protein ladder, Lane 2: RBCs treated with 1× PBS, followed by ALN, Lane 3: RBCs treated with $1 \mu \mathrm{g} / \mathrm{mL}$ PLD, followed by ALN, Lane 4: Purified His-ALN only. (B) Equal loading was ensured using Coomassie staining to determine an identical amount of erythrocytes were present for each sample.

\subsection{Treatment of Human Erythrocytes with PLD Subsequently Promotes ALN-Membrane Binding}

CDCs have long been known to follow a defined process in forming pores, starting with monomer binding to the host membrane [6,7]. Two separate regions within Domain 4 of CDCs are critical in the recognition and interaction with cholesterol: a tryptophan (Trp)-rich region known as the undecapeptide [16] and a threonine-leucine (Thr-Leu) amino acid motif [17]. The primary structure of ALN contains both an undecapeptide region (AAs 525-535) and a Thr-Leu motif (AAs 557-558) and its pore-forming activity has previously been shown to be subject to cholesterol availability within the target membrane [5]. Furthermore, point mutations within either of the motifs negatively impacts ALN-mediated hemolysis, which suggests the ability of ALN to recognize and interact with cholesterol is necessary for hemolysis (data not shown). Based on these observations, we hypothesized that by increasing the pool of ALN-accessible cholesterol, the enzymatic activity of PLD would increase the ability of ALN to bind to treated erythrocyte membranes. Erythrocytes were incubated with PLD or PBS alone, washed, and subsequently chilled and incubated with ALN to allow the CDC to bind to the erythrocyte but prevent subsequent oligomerization and pore-formation. The amount of ALN able to bind to erythrocytes was examined via Western blot. We observed a substantial increase in ALN bound to erythrocytes treated with PLD compared to erythrocytes treated with PBS alone (Figure 4). Taken in context with the data previously discussed, A. haemolyticum PLD promotes ALN-mediated hemolysis by promoting the initial binding step of the ALN pore-formation process. 


\subsection{Individual Point Mutations within PLD Decreases Sphingomyelinase Activity}

Alignments of homologous bacterial and fungal phospholipase D enzymes revealed a number of amino acids that appear to be conserved (Supplementary Materials Figure S1). We identified several amino acids within PLD as candidates for mutagenesis (P43, Y45, H49, N68, H272, and T275). Individual amino acids were mutated to an alanine as described in the Materials and Methods section and the sphingomyelinase activity of each PLD variant was evaluated. Individual point mutations were sufficient to decrease sphingomyelinase activity to 47.7-79.6\% of WT PLD levels (Figure 5).

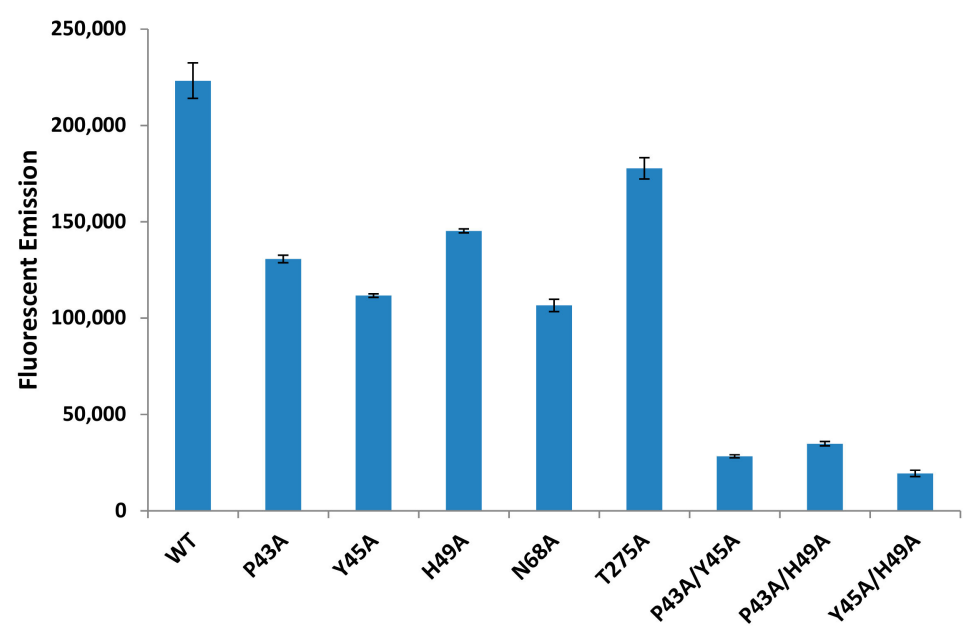

Figure 5. Mutating specific amino acids in PLD is sufficient to diminish the sphingomyelinase activity of PLD. E. coli containing a mutated pTrcHispld plasmid were grown overnight, lysed via sonication, and sphingomyelinase activity was measured using the Amplex Red Sphingomyelinase Assay kit. Bacteria containing the plasmid with one mutation possessed sphingomyelinase activity between 50 and $80 \%$ that of WT PLD while bacteria containing a plasmid with two mutations had between 8 and $15 \%$ WT PLD sphingomyelinase activity. Data is representative of three separate experiments and values represent the mean with error bars showing standard deviation. The fluorescent emission of a buffer only negative control was subtracted from each of the treatments above.

We identified the most effective single mutations and created double mutations (P43A/Y45A, P43A/H49A, and Y45A/H49A) and purified these PLD variants. All sets of double point mutations decrease sphingomyelinase activity levels to 8-15\% of WT PLD levels (Figure 5). The presence and stability of PLD variants was verified by SDS-PAGE gels stained with Coomassie Brilliant Blue staining (Data not shown).

\subsection{Sphingomyelinase-Deficient PLD Fails to Promote ALN-Mediated Hemolysis to the Same Extent as WT-PLD}

We hypothesized that the enzymatic activity of PLD was necessary to promote ALN-mediated hemolysis. Therefore, experiments outlined in Figure 3 were repeated but included the SMase-deficient PLD, with SMase activity below 10\% of WT PLD SMase activity, Y45A/H49A (Figure 6A).

Human erythrocytes were incubated with identical concentrations of WT or Y45A/H49A PLD followed by sub-hemolytic concentrations of ALN. While the WT PLD was able to promote ALN-mediated hemolysis, the mutated PLD failed to promote ALN-mediated hemolysis in neither time- (Figure 6A) nor concentration-dependent manners (Figure 6B). This observation suggests that the enzymatic activity of PLD is necessary to promote the pore-forming activity of ALN. 

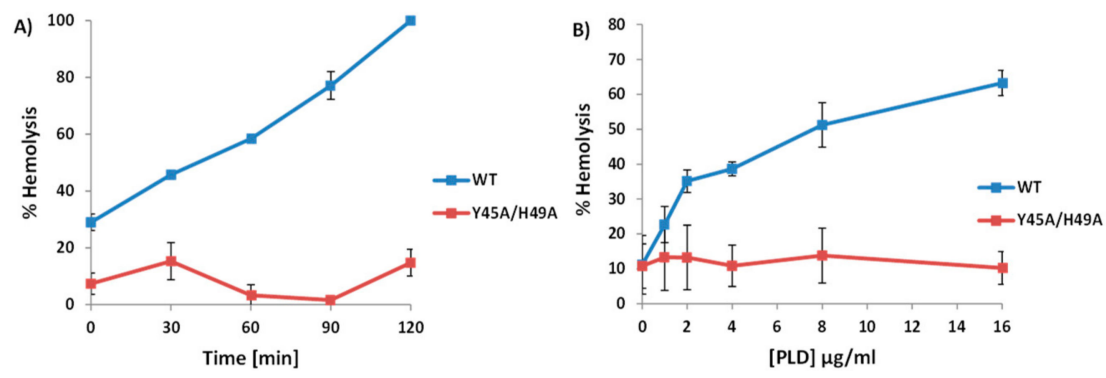

Figure 6. Sphingomyelinase-deficient PLD fails to promote ALN-mediated hemolysis. (A) $2 \mu \mathrm{g} / \mathrm{mL} \mathrm{WT}$ or Y45A/H49A PLD was incubated with 2\% human erythrocytes for between 0 and 120 min followed by the addition of $20 \mathrm{ng} / \mathrm{mL}$ ALN for an additional $30 \mathrm{~min}$ at $37^{\circ} \mathrm{C}$. (B) Varying concentrations of WT or Y45A/H49A PLD were incubated with $2 \%$ human erythrocytes for $30 \mathrm{~min}$ followed by the addition of $20 \mathrm{ng} / \mathrm{mL}$ ALN for an additional $30 \mathrm{~min}$ at $37^{\circ} \mathrm{C}$. In both cases, WT PLD increased ALN-mediated hemolysis while mutated PLD failed to promote ALN-mediated hemolysis. Each experiment was conducted three times in triplicate. Representative experiments are presented here with standard deviation represented by error bars.

\subsection{The PLD-ALN Synergistic Relationship Also Exists in a Physiologically-Relevant Cell Line}

While hemolysis assays are an effective tool to measure CDC pore-formation, it is very unlikely that erythrocytes are a biologically relevant target for ALN or PLD and there is no literature suggesting the A. haemolyticum lifecycle includes erythrocytes. Therefore, we opted to test our model in a more physiologically relevant cell line: the immortalized Detroit 562 pharyngeal epithelial cell line. Cells were seeded in a 96-well plate overnight, washed, treated with medium containing various amounts of WT or Y45A/H49A PLD followed by WT ALN or a T/L mutated ALN which fails to recognize cholesterol and has no hemolytic activity at concentrations of $100 \mu \mathrm{g} / \mathrm{mL}$ or below (Data not shown). The MTS reagent was added to each well $30 \mathrm{~min}$ after the proteins and cells had incubated to measure cell viability (Figure 7). In this assay, ALN alone was insufficient to affect cell viability but was able to decrease cell viability when added to cells pre-treated with PLD, supporting our model in a physiologically relevant cell line. In addition, when either mutated ALN or mutated PLD were substituted for their WT counterparts, no observable decrease in cell viability was observed.

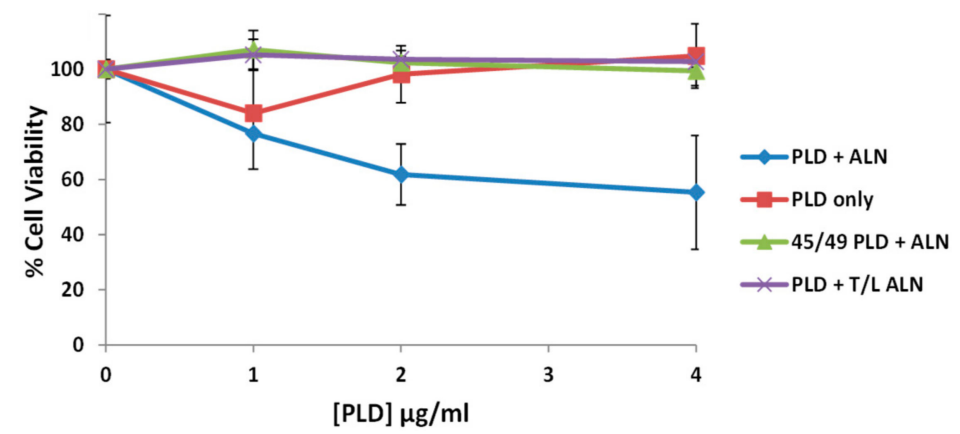

Figure 7. The PLD-ALN synergism also occurs in Detroit 562 cells. Detroit 562 cells were cultured and seeded into 96-well plates and allowed to adhere overnight. Cells were then washed with PBS and treated with medium containing 0-4 $\mathrm{gg} / \mathrm{mL}$ WT or SMase-PLD and incubated for $1 \mathrm{~h}$ at $37^{\circ} \mathrm{C}$. Cells were then washed and treated with medium containing varying concentrations of WT or T/L ALN for 30 min at $37^{\circ} \mathrm{C}$. After the incubation, all wells were treated with the MTS reagent to measure cell viability. After a 60 min incubation at $37^{\circ} \mathrm{C}$, the $\mathrm{OD}_{490}$ was measured and used to calculate the \% cell viability of cells treated with ALN alone or a combination of PLD followed by ALN. No observable decrease in cell viability was detectable when ALN only was added to the cells, but a $45 \%$ decrease in cell viability was observed when both PLD and ALN were added into the system. Each experiment was conducted three times with a representative experiment presented above. Error bars represent standard deviation. 


\section{Discussion}

A. haemolyticum is a human bacterial pathogen that has received very little attention. Since its discovery in 1946 [18], two proteins of A. haemolyticum have been identified and partially characterized: A sphingomyelin-specific PLD [8,10] and ALN [5], a member of the CDC family of pore-forming toxins. CDCs constitute a family of pore-forming toxins known to play critical roles in various aspects of bacterial lifecycles and pathogenesis [7]. A hallmark of the 50+ identified CDCs is that they appear to follow the same pore-forming choreography, but investigation of CDCs individually as well as within the context of an entire bacterial repertoire reveal characteristics unique to each CDC. Therefore, it is important to study the properties of each CDC individually as well as in the context with other bacterial factors to fully understand this unique family of pore-forming toxins and their function in bacterial lifecycles and human pathogenesis. We set forth to further our understanding of the pore-forming ability of ALN in the context of the sphingomyelinase-specific PLD from A. haemolyticum.

Like the majority of CDCs, ALN requires membrane cholesterol to mediate pore-formation [5]. The ability of ALN to interact with cholesterol can be impeded by umbrella-like covering over cholesterol from its interactions with sphingomyelin or phosphatidylcholine [11]. Many of the CDC-producing bacteria, including A. haemolyticum, produce and secrete enzymes capable of removing the head group of sphingomyelin [10], eliminating the sphingomyelin-cholesterol interaction and increasing cholesterol accessibility [11,12]. Using purified ALN and PLD, we provide evidence that the sphingomyelinase activity of PLD promotes ALN-mediated hemolysis in both time- and concentration-dependent manners. Overall, data presented in this study suggests A. haemolyticum PLD and ALN synergistically work together to form pores within a host membrane.

A key next step will be to investigate how the synergistic relationship among PLD and ALN affects interactions between $A$. haemolyticum and host epithelial cells. PLD induces the formation of lipid rafts [8], cholesterol-rich platforms that have long been hypothesized as sites of interaction between bacteria and host cells [19]. Loss of PLD was previously reported to significantly decrease A. haemolyticum adherence to and invasion of host cells in vitro [8]; however, no mechanism has been described. It has long been accepted that disturbances within plasma membranes, specifically pore-formation by CDCs, induces internalization of damaged plasma membrane [20]. It is tempting to speculate that a similar process may be hijacked by $A$. haemolyticum to induce its uptake into non-phagocytic cells.

In addition, two studies have demonstrated that $A$. haemolyticum initially resides within an unidentified vesicle during its lifecycle before entering into the host cytoplasm and inducing necrosis $[8,21]$. While the mechanism by which A. haemolyticum is initially internalized into the vesicle is unknown, $A$. haemolyticum appears to mediate its escape into the host cytoplasm in a PLD-dependent manner [8]. The lumen of an early endosome is known to be particularly enriched in sphingomyelin [22], suggesting the sphingomyelinase activity of PLD, in addition to the ALN pore-formation activity, may play an integral role during the intracellular phase of the A. haemolyticum lifecycle. Whether or not PLD and ALN work in tandem to promote the internalization of A. haemolyticum has not yet been reported, but remains an area of ongoing investigation in our laboratory.

In addition to A. haemolyticum, many CDC-producing bacteria are also known to produce and secrete phospholipases capable of acting on a variety of phospholipids including sphingomyelin, phosphatidylcholine, or phosphatidylinositol [15,23-25]. This study focused on a lipase previously shown to act only on sphingomyelin and its ability to promote the pore-forming activity of Arcanolysin. However, many CDC-producing bacteria including Clostridium perfringens, Bacillus anthracis, Bacillus cereus, and Listeria monocytogenes produce and secrete two separate phospholipases: a sphingomyelinase-specific lipase and a phosphatidylcholine-specific lipase $[15,23,24]$. In this study we also noted that the $B$. anthracis SPH enzyme also promotes ALN-mediated hemolysis, suggesting that the increase in the hemolytic activity of ALN is not increased due to an increase in a particular lipid (choline in the case of SPH or cyclic-ceramide phosphate in the case of PLD) but rather due to 
a decrease in cholesterol sequestering by sphingomyelin. Interestingly, SPH and PLD share virtually no amino acid homology, which could suggest each bacterium acquired their respective lipase and CDC by independent means. While our work demonstrates the effect sphingomyelinase activity has on ALN-mediated hemolysis, it is unknown how altering other phospholipids would affect ALN-mediated hemolysis or the hemolytic activity of other members of the cholesterol-dependent cytolysin family. Of particular interest would be phosphatidylcholine as it is also known to transiently interact with and shield cholesterol from extracellular factors (9).

Our analysis of PLD did seemingly contradict with the initial characterization of the purified enzyme. Recombinant PLD was initially reported to have no hemolytic activity [8]. However, we observed hemolysis when 20-40 $\mathrm{gg} / \mathrm{mL}$ PLD was incubated with human erythrocytes for $30 \mathrm{~min}$. The previous publication did not note the maximum concentrations used to evaluate the hemolytic activity of PLD and therefore may not have approached the minimum concentration of PLD or time period necessary to disrupt the membrane and lyse cells. The other question regarding the hemolytic activity of PLD is its mechanism of lysis. PLD is known to remove the choline head of lysophosphatidylcholine and sphingomyelin, two activities which would not be sufficient to lyse a membrane. However, there is a growing body of literature which suggests that altering the head groups of phospholipids, in particular sphingomyelin, can induce dramatic changes in membrane curvature and induce internalization of portions of a membrane [26]. It is easy to envision a point in which a critical mass of sphingomyelin within a plasma membrane has been converted into its cyclic-ceramide derivative by PLD and can no longer support the natural curvature of an intact membrane, causing lysis of the cell.

Overall, this study emphasizes the need to study the biochemistry of CDCs on an individual basis as well as in the context of other bacterial factors. Herein, we demonstrated the existence of a lipase-CDC synergistic relationship, but it is tempting to speculate on the possibility that other bacterial enzymes, such as proteases or amylases, affect membrane composition and influence the ability of CDCs to interact with and form pores within a host membrane. Understanding the interactions among multiple virulence factors and host cells may be essential to unraveling the complex and dynamic interplay between hosts and pathogens.

\section{Materials and Methods}

\subsection{Bacteria and Growth Conditions}

All A. haemolyticum strains used originated from the ATCC9345 strain. A. haemolyticum strains were grown at $37{ }^{\circ} \mathrm{C}$ with $5 \% \mathrm{CO}_{2}$ on campylobacter agar plates supplemented with $5 \%$ human blood from normal, healthy donors. A. haemolyticum strains were grown in Todd-Hewitt broth supplemented with $5 \%$ fetal bovine serum at $37{ }^{\circ} \mathrm{C}$ with $5 \% \mathrm{CO}_{2}$ with shaking. A total of $25 \mu \mathrm{g} / \mathrm{mL}$ kanamycin $(\mathrm{Kn}), 5 \mu \mathrm{g} / \mathrm{mL}$ chloramphenical $(\mathrm{Cm})$, or $10 \mu \mathrm{g} / \mathrm{mL}$ erythromycin (Er) were added to broth or agar when appropriate.

\subsection{Construction of A. haemolyticum Mutants and Complementing Plasmids}

The $\Delta$ pld and $\Delta$ pld:pld+ strains were generously provided by the Stephen Billington and Helen Jost laboratories (University of Arizona, Tucson, AZ, USA). The $\Delta a l n$ A. haemolyticum strain was constructed by PCR amplifying aln from the ATCC9345 strain of A. haemolyticum using the forward primer ( $5^{\prime}$-CGGGATCCGTCAAGTTATGCCGGGAATG-3') and reverse primer (5'-TCCGAGCTCGTTCTTGAACCAAGG-3'). The product was digested using SacI and BamHI and inserted into the intermediate vector pCRTopo creating pCRTopoaln. The aln gene was interrupted using BamHI and EcoRI enzymes with a 1.4-kb Kn resistance gene from pBS-Kan [27] digested with EcoRI. This resulted in an internal aln deletion of base pairs 656 to 763 . The resultant plasmid, pBS-aln::Kan, was electoporated into the ATCC9345 strain of A. haemolyticum and transformants were screened by PCR and Western blot to verify the presence of the interrupted aln gene and absence 
of ALN protein, respectively (data not shown). A complementing aln plasmid, pJGS182aln, was constructed by digesting both pCRTopoaln with BamHI and SacI to release a $\sim 2.0 \mathrm{~kb}$ product which included the full aln gene and promoter. The aln gene and promoter were then inserted into a pJGS182 vector digested with BamHI and SacI. pJGS182 is a pNG2 and pEP2 derivative containing ermX and an improved multi-cleaving site [28,29] The pJGS182aln plasmid was electroporated into the $\Delta a l n$ A. haemolyticum strain, conferring both ALN production and erythromycin and kanamycin resistance.

\subsection{DNA Techniques and Sequence Analysis}

Plasmid DNA extraction and transformation using E. coli DH5 $\alpha \mathrm{MCR}$ were carried out by standard methods. DNA restriction, ligation and agarose gel electrophoresis, and PCR DNA amplification were carried out as previously described [5,8].

\subsection{Purification of Recombinant His-PLD, His-ALN, His-SPH, and His-RocF}

Starting cultures of Top10F' E. coli containing either pTrcHisaln, pTrcHispld, plasmids containing a mutated aln or pld, pTrcHisB, pET-15b-antB, or pQE-rocFb were grown overnight in LB broth with $200 \mu \mathrm{g} / \mathrm{mL}$ ampicillin. Overnight cultures were diluted 1:100 in a total of $1 \mathrm{~L}$ of LB broth with ampicillin and grown at $37^{\circ} \mathrm{C}$ with shaking at $225 \mathrm{rpm}$ until the OD600 nm reached 0.6. Isopropyl $\beta$-D-1-thiogalactopyranoside (IPTG) was added to the culture to reach a final concentration of $2.5 \mathrm{mM}$ and incubated for an addition $4 \mathrm{~h}$. Cells were centrifuged at $6700 \times \mathrm{g}$ for $15 \mathrm{~min}$ at $4{ }^{\circ} \mathrm{C}$. Supernatants were removed and bacterial pellets were resuspended in $12.5 \mathrm{~mL}$ wash/lysis buffer $\left(50 \mathrm{mM} \mathrm{NaH}_{2} \mathrm{PO}_{4}, 300 \mathrm{mM} \mathrm{NaCl}, 15 \mathrm{mM}\right.$ imidazole, $\mathrm{pH}$ 8.0) and lysed using a French press twice at $16,000 \mathrm{psi}$. The remainder of the protocol was completed at $4{ }^{\circ} \mathrm{C}$. Pressed lysates were centrifuged at $9600 \times g$ and the supernatant was transferred to a fresh $50 \mathrm{~mL}$ conical tube with Ni-NTA resin (Qiagen, Carlsbad, CA, USA) at a 2:1 ratio. The supernatant/resin slurry was gently mixed end-over-end in the cold room overnight followed by transfer to sterile $5 \mathrm{~mL}$ polypropylene columns. Columns were washed 10 times with $\sim 6 \mathrm{~mL}$ wash/lysis buffer and His-tagged proteins were collected in $1 \mathrm{~mL}$ aliquots with elution buffer ( $50 \mathrm{mM} \mathrm{NaH} \mathrm{PO}_{4}, 300 \mathrm{mM} \mathrm{NaCl}, 250 \mathrm{mM}$ imidazole, $\mathrm{pH}$ 8.0). Fractions were analyzed using SDS-PAGE gels and Coomassie Blue stain to evaluate which aliquots contained protein. Elutions containing samples were combined and dialyzed in $1 \times$ PBS using Slide-A-Lyzer cassettes (ThermoFisher Scientific, Waltham, MA, USA) overnight and protein concentrations were calculated using a standard bicinchoninic acid assay (Pierce, Rockford, IL, USA).

\subsection{Hemolytic Assays}

Hemolytic activities of His-ALN and His-PLD were first determined by serially diluting each protein in $1 \times$ PBS and incubating each dilution with human erythrocytes suspended in $1 \times$ PBS. A total of $100 \mu \mathrm{L}$ of protein was incubated with $100 \mu \mathrm{L} 2 \%$ washed erythrocytes at $37^{\circ} \mathrm{C}$ for $30 \mathrm{~min}$ in 96 -well plates. Following the incubation, plates were centrifuged at $15{ }^{\circ} \mathrm{C}$ for $10 \mathrm{~min}$ at $4200 \times \mathrm{g}$ to pellet unlysed erythrocytes. Supernatants were transferred to a fresh, flat-bottom 96 -well plate and $\mathrm{OD}_{415} \mathrm{~nm}$ readings were recorded using a FLUOstar Omega plate reader.

\subsection{ALN-Binding Assays}

Whole, human blood was treated with $1 \mu \mathrm{g} / \mathrm{mL}$ PLD for $60 \mathrm{~min}$ at $37^{\circ} \mathrm{C}$ for $60 \mathrm{~min}$ with gentle agitation, washed with $1 \times$ PBS three times, and chilled on ice for $15 \mathrm{~min}$. Ice-cold, purified ALN was then added to the chilled erythrocytes for $60 \mathrm{~min}$ at $4{ }^{\circ} \mathrm{C}$ with gentle agitation to allow ALN to bind to the erythrocytes but slow the lateral diffusion and oligomerization of CDCs [30]. Samples were centrifuged at $4{ }^{\circ} \mathrm{C}$ and washed with ice-cold PBS to prevent lysis, suspended in loading buffer and run in a 10\% SDS-PAGE gel and transferred onto Immobilon PVDF Membrane (Millipore, Burlington, MA, USA) or stained with Coomassie as a loading control. Blots were blocked with $5 \%$ non-fat dry milk dissolved in Tris-buffered saline and Tween 20 (TBST; 0.5\% Tween 20) for $2 \mathrm{~h}$ at room temperature (RT). Washes between blocking, primary and secondary antibodies were performed three times for $10 \mathrm{~min}$. 
Blots were incubated with with a rabbit Anti-His-ALN primary antibody (adsorbed against E. coli containing pTrcHisB) (1:1000) (ProMab Biotechnologies, Inc., Richmond, CA, USA) and an Anti-Rabbit IgG (whole molecule) Peroxidase conjugate secondary antibody (1:10,000) (Sigma, St. Louis, MO, USA). Blots were developed with nitroblue tetrazolium/indoxyl phosphate (NBT/IP) $(100 \mu \mathrm{g} / \mathrm{mL}$ and $1 \mathrm{mg} / \mathrm{mL}$, respectively) in glycine buffer $\left(0.1 \mathrm{M}\right.$ glycine, $\left.1 \mathrm{mM} \mathrm{ZnCl}_{2}, 0.1 \% \mathrm{NaN}_{3}\right)$ at $37{ }^{\circ} \mathrm{C}$ for $30 \mathrm{~min}$.

\subsection{Sphingomyelinase Assays}

Top10F' E. coli containing the WT or mutated pTrcHispld plasmids were grown in LB broth overnight at $37^{\circ} \mathrm{C}$. Aliquots of bacteria were centrifuged for $10 \mathrm{~min}$ at $9600 \times \mathrm{g}$. Bacterial pellets were suspended in lysis buffer and sonicated for $30 \mathrm{~s}$ at $40 \%$ amplitude a total of three times using a Sonic Dismembrator 500 (Fisher Scientific, Hampton, NH, USA). Lysates were then incubated with a master mix from an Amplex Red Sphingomyelinase Assay kit (Invitrogen, Eugene, OR, USA) (200 $\mu$ M Amplex Red Reagent, $1 \mathrm{U}$ horseradish peroxidase/mL, $4 \mathrm{U}$ alkaline phosphatase/mL, $0.1 \mathrm{U}$ choline oxidase $/ \mathrm{mL}$, and $0.25 \mathrm{mM}$ sphingomyelin in $0.1 \mathrm{M}$ Tris- $\mathrm{HCl}-10 \mathrm{mM} \mathrm{MgCl}_{2}$ ). The assay was allowed to incubate at $37^{\circ} \mathrm{C}$ for 30 min protected from light. Fluorescent emissions were measured using a FLUOstar Omega plate reader with excitation at $544 \mathrm{~nm}$ and detection at $590 \mathrm{~nm}$.

\subsection{Mutagenesis of Pld or Aln}

Point mutations were engineered into pTrcHispld or pTrcHisaln using the QuikChange XL Site-Directed Mutagenesis kit (Agilent Technologies, Cedar Creek, TX, USA). Briefly, PCR products originating from pTrcHispld or pTrcHisaln and corresponding primers (Table 1) were incubated with DpnI and heat shocked into Top10F' E. coli and grown overnight on LB plates supplemented with ampicillin. Transformants were selected and SMase activity levels of E. coli lysates were measured using the Amplex Red Sphingomyelinase Assay kit. Plasmids from transformants with decreased SMase levels were sequenced (Arizona State University DNA Sequencing Lab, Tempe, AZ, USA) to confirm the presence of the mutation.

Table 1. List of primers used in this study.

\begin{tabular}{cr}
\hline Primer Name & Sequence (5' -3') $\left.^{\prime}\right)$ \\
\hline DM1117 (T557A_Forward) & CGCGAGATCATCCTTCGCGGTACTGCCTTACGGCCTTGGTTC \\
DM1118 (T557A_Reverse) & GAACCAAGGCCGTAAGGCAGTACCGCGAAGGATGATCTCGCG \\
DM1121 (L558A_Forward) & CGCGAGATCATCCTTCGCGGTACTACCGCACGGCCTTGGTTC \\
DM1122 (L558A_Reverse) & GAACCAAGGCCGTGCGGTAGTACCGCGAAGGATGATCTCGCG \\
DM1125 (TL55758AA_Forward) & CGCGAGATCATCCTTCGCGGTACTGCGGCACGGCCTTGGTTC \\
DM1126 (TL557_58AA_Reverse) & GAACCAAGGCCGTGCCGCAGTACCGCGAAGGATGATCTCGCG \\
DM1162 (P43A_Forward) & ACCAACCACTGGTAACCGTGCCGTCTATGCCATTGCGCACC \\
DM1163 (P43A_Reverse) & GGTGCGCAATGGCATAGACGGCACGGTTACCAGTGGTTGGT \\
DM1164 (Y45A_Forward) & AGAACACGGTGCGCAATGGCGGCGACTGGACGGTTACCAGTGG \\
DM1165 (Y45A_Reverse) & CCACTGGTAACCGTCCAGTCGCCGCCATTGCGCACCGTGTTCT \\
DM1166(H49A_Forward) & TTTGCTTCGTCAGAACACGGGCCGCAATGGCATAGACTGGAC \\
DM1167(H49A_Reverse) & GTCCAGTCTATGCCATTGCGGCCCGTGTTCTGACGAAGCAAA \\
DM1168(N68A_Forward) & AGTAAAATCAATTTCCAGAGCGGCCGCGCCAATTTTGATTGCGTC \\
DM1169(N68A_Reverse) & GACGCAATCAAAATTGGCGCGGCCGCTCTGGAAATTGATTTTACT \\
DM1170(H272A_Forward) & AATGTCTTTGTTGGTTGCCATGGCGTGAGTACCTTGATGTGCATC \\
DM1171(H272A_Reverse) & GGATGCACATCAAGGTACTCACGCCATGGCAACCAACAAAGACAT \\
DM1172(T275A_Forward) & ACGGAATGTCTTTGTTGGCTGCCATGTGGTGAGTACCTTG \\
DM1173(T275A_Reverse) & CAAGGTACTCACCACATGGCAGCCAACAAAGACATTCCGT \\
DM1174 (P43A,Y45A_F) & CGGTGCGCAATGGCGGCGACGGCACGGTTACCAGTGGTTGGTTGTTCTT \\
DM1175 (P43A,Y45A_R) & AAGAACAACCAACCACTGGTAACCGTGCCGTCGCCGCCATTGCGCACCG \\
DM1176 (P43A,H49A_F) & CTTTGCTTCGTCAGAACACGGGCCGCAATGGCATAGACGGCACGG \\
DM1177 (P43A,H49A_R) & CCGTGCCGTCTATGCCATTGCGGCCCGTGTTCTGACGAAGCAAAG \\
DM1178 (Y45A,H49A_F) & GCTTCGTCAGAACACGGGCCGCAATGGCGGCGACTGGACGGTTACCAG \\
DM1179 (Y45A,H49A_R) & CTGGTAACCGTCCAGTCGCCGCCATTGCGGCCCGTGTTCTGACGAAGC \\
\hline
\end{tabular}




\subsection{Cell Viability Assays}

Detroit 562 cells were grown in F-12 Ham's Medium supplemented with 10\% FBS. Approximately $1 \times 10^{4}$ cells were seeded in a Costar 96-well flat bottom plate and allowed to adhere overnight. Cells were then washed with with $1 \times$ PBS a total of three times and the medium was replaced with F-12 Ham's Medium alone (lacking FBS) containing various concentrations of PLD and incubated for $1 \mathrm{~h}$ at $37^{\circ} \mathrm{C}$. Cells were then washed and the medium was replaced with F-12 Ham's Medium (no FBS) containing various concentrations of ALN. Cells were allowed to incubate for $30 \mathrm{~min}$ at $37^{\circ} \mathrm{C}$, at which point $20 \mu \mathrm{L}$ of MTS Reagent (Biovision Incorporated, Milpitas, CA, USA) was added to each well. After the MTS Reagent had been added to the cells for $60 \mathrm{~min}, \mathrm{OD}_{490} \mathrm{~nm}$ readings were taken and, with a medium-only negative control and Trition X-100 positive control, used to calculate cell viability.

Supplementary Materials: The following are available online at http:/ /www.mdpi.com/2072-6651/10/6/213/s1, Figure S1: Sequence alignment of various PLD amino acid sequences.

Author Contributions: Conceptualization, P.S.G. and D.J.M.; Methodology, P.S.G.; Investigation, P.S.G.; Formal Analysis, P.S.G. and D.J.M.; Resources, D.J.M.; Supervision, D.J.M.; Visualization, P.S.G. and D.J.M.; Funding acquisition, P.S.G. and D.J.M.; Writing-Original Draft Preparation, P.S.G.; Writing-Review \& Editing, P.S.G. and D.J.M.

Acknowledgments: We appreciate the helpful discussions with Adam J. Ratner (NYU Langone Health), Haley S. Ruther-Norwood (Cedars-Sinai Medical Center) for construction of the $\Delta$ aln A. haemolyticum strain, the Andrew D. Yurochko laboratory for providing human erythrocytes, and Stephen Leppla for generously providing the pET-15b-antB plasmid used in purifying B. anthracis SPH.

Conflicts of Interest: The authors declare no conflict of interest.

\section{References}

1. Linder, R. Rhodococcus equi and Arcanobacterium haemolyticum: Two "coryneform" bacteria increasingly recognized as agents of human infection. Emerg. Infect. Dis. 1997, 3, 145-153. [CrossRef] [PubMed]

2. Banck, G.; Nyman, M. Tonsillitis and rash associated with Corynebacterium haemolyticum. J. Infect. Dis. 1986, 154, 1037-1040. [CrossRef] [PubMed]

3. Mackenzie, A.; Fuite, L.A.; Chan, F.T.; King, J.; Allen, U.; MacDonald, N.; Diaz-Mitoma, F. Incidence and pathogenicity of Arcanobacterium haemolyticum during a 2-year study in Ottawa. Clin. Infect. Dis. Off. Publ. Infect. Dis. Soc. Am. 1995, 21, 177-181. [CrossRef]

4. Miller, R.A.; Brancato, F.; Holmes, K.K. Corynebacterium hemolyticum as a cause of pharyngitis and scarlatiniform rash in young adults. Ann. Intern. Med. 1986, 105, 867-872. [CrossRef] [PubMed]

5. Jost, B.H.; Lucas, E.A.; Billington, S.J.; Ratner, A.J.; McGee, D.J. Arcanolysin is a cholesterol-dependent cytolysin of the human pathogen Arcanobacterium haemolyticum. BMC Microbiol. 2011, 11, 239. [CrossRef] [PubMed]

6. Tweten, R.K.; Hotze, E.M.; Wade, K.R. The unique molecular choreography of giant pore formation by the cholesterol-dependent cytolysins of Gram-Positive bacteria. Annu. Rev. Microbiol. 2015, 69, 323-340. [CrossRef] [PubMed]

7. Tweten, R.K. Cholesterol-dependent cytolysins, a family of versatile pore-forming toxins. Infect. Immun. 2005, 73, 6199-6209. [CrossRef] [PubMed]

8. Lucas, E.A.; Billington, S.J.; Carlson, P.; McGee, D.J.; Jost, B.H. Phospholipase D promotes Arcanobacterium haemolyticum adhesion via lipid raft remodeling and host cell death following bacterial invasion. BMC Microbiol. 2010, 10, 270. [CrossRef] [PubMed]

9. Selvy, P.E.; Lavieri, R.R.; Lindsley, C.W.; Brown, H.A. Phospholipase D: Enzymology, functionality, and chemical modulation. Chem. Rev. 2011, 111, 6064-6119. [CrossRef] [PubMed]

10. Lajoie, D.M.; Cordes, M.H.J. Spider, bacterial and fungal phospholipase D toxins make cyclic phosphate products. Toxicon Off. J. Int. Soc. Toxinol. 2015, 108, 176-180. [CrossRef] [PubMed]

11. Fantini, J.; Barrantes, F.J. How cholesterol interacts with membrane proteins: An exploration of cholesterol-binding sites including CRAC, CARC, and tilted domains. Front. Physiol. 2013, 4, 31. [CrossRef] [PubMed] 
12. Das, A.; Brown, M.S.; Anderson, D.D.; Goldstein, J.L.; Radhakrishnan, A. Three pools of plasma membrane cholesterol and their relation to cholesterol homeostasis. eLife 2014, 3, e02882. [CrossRef] [PubMed]

13. McGee, D.J.; Zabaleta, J.; Viator, R.J.; Testerman, T.L.; Ochoa, A.C.; Mendz, G.L. Purification and characterization of Helicobacter pylori arginase, RocF: Unique features among the arginase superfamily. Eur. J. Biochem. 2004, 271, 1952-1962. [CrossRef] [PubMed]

14. García-Arribas, A.B.; Alonso, A.; Goñi, F.M. Cholesterol interactions with ceramide and sphingomyelin. Chem. Phys. Lipids 2016, 199, 26-34. [CrossRef] [PubMed]

15. Pomerantsev, A.P.; Kalnin, K.V.; Osorio, M.; Leppla, S.H. Phosphatidylcholine-specific phospholipase C and sphingomyelinase activities in bacteria of the Bacillus cereus group. Infect. Immun. 2003, 71, 6591-6606. [CrossRef] [PubMed]

16. Dowd, K.J.; Farrand, A.J.; Tweten, R.K. The cholesterol-dependent cytolysin signature motif: A critical element in the allosteric pathway that couples membrane binding to pore assembly. PLoS Pathog. 2012, 8, e1002787. [CrossRef]

17. Farrand, A.J.; LaChapelle, S.; Hotze, E.M.; Johnson, A.E.; Tweten, R.K. Only two amino acids are essential for cytolytic toxin recognition of cholesterol at the membrane surface. Proc. Natl. Acad. Sci. USA 2010, 107, 4341-4346. [CrossRef] [PubMed]

18. Maclean, P.D.; Liebow, A.A.; Rosenberg, A.A. A hemolytic corynebacterium resembling Corynebacterium ovis and Corynebacterium pyogenes in man. J. Infect. Dis. 1946, 79, 69-90. [CrossRef] [PubMed]

19. Zaas, D.W.; Duncan, M.; Rae Wright, J.; Abraham, S.N. The role of lipid rafts in the pathogenesis of bacterial infections. Biochim. Biophys. Acta 2005, 1746, 305-313. [CrossRef] [PubMed]

20. Corrotte, M.; Fernandes, M.C.; Tam, C.; Andrews, N.W. Toxin Pores Endocytosed during Plasma Membrane Repair Traffic into the Lumen of MVBs for Degradation: Endocytosed SLO Pores are Degraded in Lysosomes. Traffic 2012, 13, 483-494. [CrossRef] [PubMed]

21. Osterlund, A. Are penicillin treatment failures in Arcanobacterium haemolyticum pharyngotonsillitis caused by intracellularly residing bacteria? Scand. J. Infect. Dis. 1995, 27, 131-134. [CrossRef] [PubMed]

22. Hullin-Matsuda, F.; Taguchi, T.; Greimel, P.; Kobayashi, T. Lipid compartmentalization in the endosome system. Semin. Cell Dev. Biol. 2014, 31, 48-56. [CrossRef] [PubMed]

23. O'Brien, D.K.; Melville, S.B. Effects of Clostridium perfringens alpha-toxin (PLC) and perfringolysin O (PFO) on cytotoxicity to macrophages, on escape from the phagosomes of macrophages, and on persistence of $C$. perfringens in host tissues. Infect. Immun. 2004, 72, 5204-5215. [CrossRef] [PubMed]

24. Schlüter, D.; Domann, E.; Buck, C.; Hain, T.; Hof, H.; Chakraborty, T.; Deckert-Schlüter, M. Phosphatidylcholine-specific phospholipase $\mathrm{C}$ from Listeria monocytogenes is an important virulence factor in murine cerebral listeriosis. Infect. Immun. 1998, 66, 5930-5938. [PubMed]

25. Moe, P.C.; Heuck, A.P. Phospholipid hydrolysis caused by Clostridium perfringens $\alpha$-toxin facilitates the targeting of perfringolysin O to membrane bilayers. Biochemistry (Mosc.) 2010, 49, 9498-9507. [CrossRef] [PubMed]

26. Dinkla, S.; Wessels, K.; Verdurmen, W.P.R.; Tomelleri, C.; Cluitmans, J.C.A.; Fransen, J.; Fuchs, B.; Schiller, J.; Joosten, I.; Brock, R.; et al. Functional consequences of sphingomyelinase-induced changes in erythrocyte membrane structure. Cell Death Dis. 2012, 3, e410. [CrossRef] [PubMed]

27. Langford, M.L.; Zabaleta, J.; Ochoa, A.C.; Testerman, T.L.; McGee, D.J. In vitro and in vivo complementation of the Helicobacter pylori arginase mutant using an intergenic chromosomal site. Helicobacter 2006, 11, 477-493. [CrossRef] [PubMed]

28. Radford, A.J.; Hodgson, A.L. Construction and characterization of a Mycobacterium-Escherichia coli shuttle vector. Plasmid 1991, 25, 149-153. [CrossRef]

29. Messerotti, L.J.; Radford, A.J.; Hodgson, A.L. Nucleotide sequence of the replication region from the Mycobacterium-Escherichia coli shuttle vector pEP2. Gene 1990, 96, 147-148. [CrossRef]

30. Gekara, N.O.; Jacobs, T.; Chakraborty, T.; Weiss, S. The cholesterol-dependent cytolysin listeriolysin O aggregates rafts via oligomerization: Aggregation of lipid rafts by listeriolysin O. Cell. Microbiol. 2005, 7, 1345-1356. [CrossRef] [PubMed]

(C) 2018 by the authors. Licensee MDPI, Basel, Switzerland. This article is an open access article distributed under the terms and conditions of the Creative Commons Attribution (CC BY) license (http://creativecommons.org/licenses/by/4.0/). 